\title{
A Bayesian Approach for the Cox Proportional Hazards Model with Covariates Subject to Detection Limit
}

\author{
Qingxia Chen ${ }^{1,2,}$, Huiyun $\mathrm{Wu}^{3}$, Lorraine B. Ware ${ }^{4}$ and Tatsuki Koyama ${ }^{1}$ \\ ${ }^{1}$ Department of Biostatistics, Vanderbilt University, Nashville, Tennessee, 37232, USA \\ ${ }^{2}$ Department of Biomedical Informatics, Vanderbilt University, Nashville, Tennessee, 37232, USA \\ ${ }^{3}$ Department of Biostatistics, St. Jude Children's Research Hospital, Memphis, Tennessee, 38105, USA \\ ${ }^{4}$ Department of Medicine, Vanderbilt University, Nashville, Tennessee, 37232, USA
}

\begin{abstract}
The research on biomarkers has been limited in its effectiveness because biomarker levels can only be measured within the thresholds of assays and laboratory instruments, a challenge referred to as a detection limit (DL) problem. In this paper, we propose a Bayesian approach to the Cox proportional hazards model with explanatory variables subject to lower, upper, or interval DLs. We demonstrate that by formulating the time-to-event outcome using the Poisson density with counting process notation, implementing the proposed approach in the OpenBUGS and JAGS is straightforward. We have conducted extensive simulations to compare the proposed Bayesian approach to the other four commonly used methods and to evaluate its robustness with respect to the distribution assumption of the biomarkers. The proposed Bayesian approach and other methods were applied to an acute lung injury study, in which a panel of cytokine biomarkers was studied for the biomarkers' association with ventilation-free survival.
\end{abstract}

Keywords: Bayesian, Biomarker, Detection limit, Lung Injury, Proportional hazards models.

\section{INTRODUCTION}

Biomarkers have been increasingly and widely used in clinical practice in recent years for disease diagnosis and prognosis based on their underlying pathological and physiologic mechanisms [1]. In these applications, biomarkers are used either to identify a subgroup of a study population or predict a disease outcome [2]. While some biomarkers are involved in the early development of a condition and thus might provide diagnoses [3, 4], others are associated more with disease outcome and are considered prognostic of patient survival [5]. For example, a nationwide cohort study revealed that a traditional serum biomarker leukotriene pathway agent was associated with the breast cancer; p53 expression in primary tumors was an independent prognostic factor that influenced relapse-free survival in stage II patients, and lack of $\mathrm{Bcl}-2$ expression was independently associated with a poor prognosis among stage III patients [6]. Another large cohort study identified protein biomarker CTNNB1 to be associated with improved survival in colorectal cancer [3]. Discrepancies between studies on the same biomarkers have been observed [7], however, and a variety of problems have been cited as the cause of these discrepancies including inappropriate statistical analyses [8]. As a result, in 2005, REporting recommendations for tumor MARKer

*Address correspondence to this author at the Department of Biostatistics, Vanderbilt University, Nashville, Tennessee, 37232, USA; Tel: +00-615-9368058; Fax: +00-615-343-4924; E-mail: cindy.chen@vanderbilt.edu prognostic studies (RE-MARK) was published [7]. One of the goals of these guidelines was to improve the usefulness of the results from clinical prognostic studies and enhance the comparability between studies.

Detection limit (DL) is a measurement error problem with bounded error $[9,10]$. In particular, $D L$ is a measurement problem in which the actual biomarker values are immeasurable either below the lower detection limit (LDL) or above the upper detection limit (UDL) of laboratory instruments. These values are often called non-detects. The applicability of biomarkers in clinical practice has been compromised in the presence of $\mathrm{DL}$ as inappropriate use of statistical methods when dealing with $\mathrm{DL}$ may lead to biased conclusions and inconsistent results [11]. Simple methods, such as deletion and single replacement with one-half of the LDL, were often used to fill in the immeasurable values. Some sophisticated methods have been proposed to replace the missing values based on the distribution of the observed values. Regression on Order Statistic (ROS) is one such approach; however, its usage is severely limited because its goal is to estimate summary statistics [12]. More recently, vast researches have been conducted in $\mathrm{DL}$ and its related areas. For example, an MLE-based approach has been proposed to handle both binary [13] and continuous outcomes [14] with an independent variable subject to DL; a semiparametric Bayesian method was proposed under proportional hazards model for interval censored data with frailty effects [15]; a semiparametric imputation approach has 
been developed for covariates subject to $\mathrm{DL}$, in which the conditional quantiles of the censored covariates are assumed to be linear in the observed variables; $[16,17]$ have proposed a Bayesian approach to logistic regression parameter estimation with exposure variables subject to DL; [18] discussed the full Bayesian estimation of joint models when time dependent covariates or outcomes are submitted to lower detection levels. These works have improved the usefulness of biomarkers in the development of diagnostic and prognostic models. However, in general there remain a few problems to be solved. To our knowledge, many of the medical researches involving Cox proportional hazards models with DL biomarkers still relied on simple naïve methods like deletion or single replacement to obtain the estimates of hazard ratio $(\mathrm{HR})$, mainly due to the computational burden of advanced methods and lacking of user-accessible programs. Given promising prognostic value and increased clinical application of biomarkers in disease outcome prediction, a survival model that is capable of handling $\mathrm{DL}$ is desired. In this paper, we formulated the time-to-event outcome using the Poisson density with counting process notation, and provided straightforward JAGS/OpenBUGS programs to implement the method. In the current clinical practice, a panel of biomarkers, rather than a single biomarker, is often considered for disease diagnosis/prognosis [19], and therefore, we aim to develop a method to handle simultaneous $\mathrm{DL}$ issues on multiple biomarkers.

The paper is organized as follows. In the Motivating Study Section, we provide a detailed description of the motivating study which illustrates the association between biomarkers and the survival function for patients with acute lung injury (ALI). In the Methodological Development Section, we describe the proposed Bayesian method for the cases in which single or multiple explanatory variables are subject to lower, upper, and interval DLs. In the Simulation Studies Section, we present an extensive simulation study to examine the performance of the proposed method, comparing it with four existing methods. We revisit the motivating study in the section of Analysis of the Acute Lung Injury Study, followed by the Discussion Section.

\section{MOTIVATING STUDY}

With the goal of developing a prognostic model for Acute Lung Injury/Acute Respiratory Distress Syndrome (ALI/ARDS), the researcher measured 8 cytokine biomarkers that reflect the complex pathogenesis of ALI/ARDS in baseline plasma from 549 patients [20]. The patients were enrolled in the National Heart, Lung, and Blood Institute (NHLBI) ARDS Clinical Trials Network clinical trial of two different levels of positive end-expiratory pressure [20]. The collected biomarkers included markers of inflammation (IL6, IL8, and TNFR1), lung and systemic endothelial activation and injury (VWF), lung epithelial injury (SP-D), adhesion molecules (ICAM.1), and activation of coagulation and inhibition of fibrinolysis (protein $\mathrm{C}$, and PAI-1). Among these biomarkers, IL8 had $35 \%$ of values that were below the DL threshold of $2.5 \mathrm{pg} / \mathrm{ml}$ of the enzyme-linked immunosorbent assay. Collected clinical data include age, cause of ALI/ARDS, severity of illness scoring (APACHE III), ventilator parameters, hemodynamic data, and alveolar-arterial $\mathrm{O}_{2}$ difference $\left(\begin{array}{lll}A-a & O_{2}\end{array}\right)$. Cox proportional hazards models were used to investigate the association between the biomarker levels and time to ventilation removal (VR).

\section{METHODOLOGICAL DEVELOPMENT}

Let $T_{i}$ and $C_{i}$ represent the failure and censoring times, respectively, for the ith patient, where $i=1, \ldots, n$. Let $X_{i}$ be the transformed biomarker covariates subject to detection limit with a known transformation function $g(\cdot)$ and $Z_{i}$ be the additional covariates.

Observed times to event are denoted by $Y_{i}=T_{i} \wedge C_{i}$, and the observed event indicators are denoted by $\Delta_{i}=I\left(T_{i} \leq C_{i}\right)$ where $a \wedge b=\min (a, b)$ and $I(A)$ is an indicator function taking the value 1 when condition $\mathrm{A}$ holds and 0 otherwise.

In this paper, we consider the Cox proportional hazards model [21] given by

$$
\lambda_{i}\left(t \mid Z_{i}, X_{i}\right)=\lambda_{0}(t) \exp \left(\beta^{T} X_{i}+\gamma^{T} Z_{i}\right) d t
$$

where $\lambda(t \mid Z, X)$ is the conditional hazard function of time-to-event given the covariates, $\lambda_{0}(t)$ is an unknown baseline function, $\beta$ is a $q \times 1$ vector of regression coefficients corresponding to transformed biomarker covariates $X_{i}$, and $\gamma$ is a $\mathrm{p} \times 1$ vector of regression coefficients for other additional covariates $Z_{i}$.

\section{Single Covariate Subject to DL}

We first consider the case of one covariate subject to $D L(q=1)$ and then generalize this method to the scenario with multiple covariates subject to $D L(q>1)$ in 
the next section. With $q=1$, we assume the lower $D L$ for the transformed biomarker measurement $\mathrm{x}$ is $\mathrm{ld}$. The observed data are

$D_{o b s}=\left(\gamma_{i}, \delta_{i}, x_{i}^{*}=\left(x_{i} \mathrm{vld}\right), r_{i}=I\left(x_{i} \leq l d\right), z_{i}, i=1,2, \cdots, n\right)$,

where $a \vee b=\max (a, b)$. The likelihood function of the observed data is

$$
\begin{aligned}
& L=\prod_{i=1}^{n}\left[L y_{i}\left(y_{i} \mid x_{i}, z_{i}, \beta, \gamma\right) f\left(x_{i}^{*} \mid z_{i}, \phi\right)\right]^{\left(1-r_{i}\right)} \\
& {\left[\int_{g(0)}^{l d} L y_{i}\left(y_{i} \mid x, z_{i}, \beta, \gamma\right) f\left(x \mid z_{i}, \phi\right) d x\right]^{r_{i}}}
\end{aligned}
$$

where $L y_{i}(\cdot)$ is the likelihood of $\left(y_{i}, \delta_{i}\right)$ and $\phi$ is a vector of regression coefficients in the biomarker model $f\left(x_{i}^{*} \mid z_{i}, \phi\right)$, both will be discussed later. Note that the lower limit of the integration in (2) is $g(0)$ because biomarker measurements have support $(0, \infty)$, and $\mathrm{x}$ is the transformed biomarker measurement with transformation function $\mathrm{g}(\cdot)$.

Extending the proposed method to interval DL (both lower and upper DLs) is straightforward. We assume the upper DL for $\mathrm{x}$ is ud and denote the observations as

$D_{o b s}=\left(y_{i}, \delta_{i}, x_{i}^{*}=\left(x_{i} \vee \mathrm{ld} \wedge \mathrm{ud}\right), r_{i l}=I\left(x_{i} \leq l d\right), r_{i u}\right.$

$\left.=I\left(x_{i} \geq u d\right), z_{i}, i=1,2, \cdots, n\right)$

The likelihood function of the observed data for the th subject now becomes

$$
\begin{aligned}
& L i=\prod_{i=1}^{n}\left[L y_{i}\left(y_{i} \mid x_{i}, z_{i}, \beta, \gamma\right) f\left(x_{i}^{*} \mid z_{i}, \phi\right)\right]^{\left(1-r_{i i}\right)\left(1-r_{i u}\right)} \\
& {\left[\int_{g(0)}^{l d} L y_{i}\left(y_{i} \mid x, z_{i}, \beta, \gamma\right) f\left(x \mid z_{i}, \phi\right) d x\right]^{r_{i t}\left(1-r_{i u}\right)}} \\
& {\left[\int_{u d}^{g(\infty)} L y_{i}\left(y_{i} \mid x, z_{i}, \beta, \gamma\right) f\left(x \mid z_{i}, \phi\right) d x\right]^{\left(1-r_{i l}\right) r_{i u}}}
\end{aligned}
$$

\section{Multiple Covariates Subject to DL}

When there are two or more covariates subject to $D L, f(x \mid z)$ in (2) becomes a multivariate density function. A simple extension may be to model $f(x \mid z)$ through a multivariate Gaussian distribution; however, the normality assumption may not hold for all biomarkers subject to DL. Therefore, following [22, 23], we model the joint distribution of the biomarkers using a series of one dimensional conditional densities as

$$
\begin{aligned}
& f(x \mid z, \phi)=f\left(x_{i 1} \mid z_{i}, x_{i 2}, \cdots, x_{i q}, \phi_{1}\right) f\left(x_{i 2} \mid z_{i}, x_{i 3}, \cdots, x_{i 3}, \cdots, x_{i q}, \phi_{2}\right) \\
& \cdots f\left(x_{i q} \mid z_{i}, \phi_{q}\right),
\end{aligned}
$$

where $\phi_{k}$ is the vector of unknown parameters in the distribution of $f\left(x_{i k} \mid z_{i}, x_{i, k+1}, \cdots, x_{i q}, \phi_{k}\right), \quad$ and $\phi=\left(\phi_{1}, \cdots, \phi_{q}\right)$. The advantage of (4) is that it allows for a more flexible model specification for the joint distribution of $f(x \mid z)$, and it is especially useful when the biomarkers follow different distribution functions. Although the joint model depends on the order of conditioning, [22, 23], among others, have shown in the missing data literature that estimates are robust with respect to the order of conditioning; however, by no means should one discount the importance of carrying out sensitivity analysis for the order of conditioning.

When there are $\mathrm{q}$ biomarkers subject to lower $\mathrm{DL}$, the observed data are

$$
\begin{aligned}
& D_{o b s}=\left(y_{i}, \delta_{i}, x_{i 1}^{*}=\left(x_{i 1} \vee l d_{1}\right), r_{i 1}=I\left(x_{i 1} \leq l d_{1}\right), \cdots, x_{i q}^{*}=\left(x_{i q} \vee l d_{q}\right),\right. \\
& \left.r_{i q}=I\left(x_{i q} \leq l d\right), z_{i}, i=1,2, \cdots, n\right),
\end{aligned}
$$

where $l d_{k}$ is the lower DL of $x_{k}$. Let $x_{i,-j}=\left(x_{i 1}, \cdots, x_{i, j-1}, x_{i, j+1}, \cdots, x_{i q}\right)$ denote all the biomarkers from the ith subject except the jth biomarker. The likelihood function of the ith observed value is

$$
\begin{aligned}
& L_{i}=\left[L y_{i}\left(y_{i} \mid x_{i}=x_{i}^{*}, z_{i}, \beta, \gamma\right) f\left(x_{i}^{*} \mid z_{i}, \phi\right)\right]^{\left(1-r_{i 1}\right) \cdots\left(1-r_{i q}\right)} \\
& \prod_{j=1}^{q}\left[\int_{g(0)}^{l d_{j}} L y_{i}\left(y_{i} \mid x_{i,-j}, x, z_{i}, \beta, \gamma\right) f\left(x_{i, j-1}, x \mid z_{i}, \phi\right) d x\right]^{\left(1-r_{i 1}\right) \cdots r_{i j} \cdots\left(1-r_{i q}\right)} \\
& \ldots\left[\int_{g(0)}^{l d_{q}} \cdots \int_{g(0)}^{l d_{1}} L y_{i}\left(y_{i} \mid x, z_{i}, \beta, \gamma\right) f\left(x \mid z_{i}, \phi\right) d x_{1} \cdots d x_{q}\right]^{r_{i 1} \cdots r_{i q}}
\end{aligned}
$$

where $f\left(\boldsymbol{x}_{\boldsymbol{i}} \mid z_{i}, \boldsymbol{\phi}\right)$ is defined in (4).

\section{Counting Process Likelihood for Posterior Computation and Inference}

For purposes of computation and ease of extending the proposed method, we will formulate the likelihood of $\left(y_{i}, \delta_{i}\right)$ using the counting process in this section. In particular, for subject $i$, we observe counting process $N_{i}(t)$, which counts the number of failures occurred up to time $t$. Following [24], the Cox proportional hazards model in (1) can be formulated using the counting process notation introduced by [25] and is given by

$$
E\left\{d N_{i}(t) \mid \mathcal{F}_{t-}, \boldsymbol{Z}, \boldsymbol{X}\right\}=\lambda_{0}(t) \exp \left(\boldsymbol{\beta}^{T} \boldsymbol{X}_{\boldsymbol{i}}+\boldsymbol{\gamma}^{T} \boldsymbol{Z}_{\boldsymbol{i}}\right) d t
$$


where $\mathcal{F}_{t \text { - }}$ represents all the past history just before time $\mathrm{t}$, and $d N_{i}(t)$ is the increment of $N_{i}(t)$ over the small time interval [t, $\mathrm{t}+\mathrm{dt}$ ]; $d N_{i}(t)=1$ if subject $\mathrm{i}$ is observed to fail during the time interval $[\mathrm{t}, \mathrm{t}+\mathrm{dt}]$, and $d N_{i}(t)=0$ otherwise. The observed data from $\mathrm{n}$ subjects can be rewritten using the counting process given by $\left\{N_{i}(t) I\left(t \leq Y_{i}\right), Y_{i}, \boldsymbol{R}_{i}, Z_{i}, C_{i}\right\}, i=1,2, \cdots, n$ where $\boldsymbol{X}_{i}^{*}=\left(\boldsymbol{x}_{i 1}^{*}, \cdots, \boldsymbol{x}_{i q}^{*}\right) \quad$ and $\quad \boldsymbol{R}_{i}=\left(r_{i 1}, \cdots \boldsymbol{r}_{i q}\right)$. Under the standard non-informative censoring assumption, the counting process likelihood of $\left\{N_{i}(t) I\left(t \leq Y_{i}\right), Y_{i}\right\}$ given $\left(X, Z_{i}, C_{i}\right)$ is

$L y_{i}=\left\{\prod_{t \leq Y_{i}} \lambda_{i}(t)^{d N_{i}(t)}\right\} \exp \left\{-\int_{0}^{Y_{i}} \lambda_{i}(t) d t\right\}$

where $\lambda_{i}(t)=\lambda_{0}(t) \exp \left(\beta^{T} X_{i}+\gamma^{T} Z_{i}\right) d t$.

Following [24], we can express the counting process likelihood in (7) using Poisson density as

$$
L_{y i}=\prod_{j=1}^{J} \operatorname{Poisson}\left(d N_{i j} ; V_{i j} d \Lambda_{0 J} \exp \left(\boldsymbol{\beta}^{T} \boldsymbol{X}_{i}+\boldsymbol{\gamma}^{T} \boldsymbol{Z}_{i}\right)\right)
$$

where the notation Poisson $(x ; \mu)$ is the density of a random variable $x$, which follows a Poisson distribution with mean $\mu ; t_{1}, \cdots, t_{J}$ are the unique failure times observed in the data, $V_{i j}=1$ if subject $\mathrm{i}$ is at risk at time $t_{i}$ and $V_{i j}=0$ otherwise, $d N_{i j}=1$ if individual $i$ fails at time $t_{i}$ and $d N_{i j}=0$ otherwise, and $d \Lambda_{0 j}$ is an increment on the cumulative baseline hazard function $\Lambda_{0}(t)=\int_{0}^{t} \lambda_{0}(\mu) d \mu$.

The joint posterior density of $\left(\beta, \gamma, d \Lambda_{0}(\cdot), \phi\right)$ based on the observed data can be written as

$\pi\left(\beta, \gamma, d \Lambda_{0}(\cdot), \phi \mid D_{o b s}\right) \propto L \times \pi\left(\beta, \gamma, d \Lambda_{0}(\cdot), \phi\right)$,

where $L=\prod_{i=1}^{n} L_{i}, L_{i}$ is defined in (3) or (5) for the univariate or multivariate biomarkers subject $\mathrm{DL}$, respectively, $L_{y i}$ is defined in (8), and $\pi(\beta, \gamma, \mathrm{d} \wedge 0(\cdot)$, $\phi)$ is the joint prior for $(\beta, \gamma, \mathrm{d} \wedge 0(\cdot), \phi)$. In practice, we can specify the independent prior and let $\pi(\beta, \gamma$, $\mathrm{d} \wedge 0(\cdot), \phi)=\pi(\beta) \pi(\gamma) \pi(\mathrm{d} \wedge 0(\cdot)) \pi(\phi)$. We can specify the non-informative prior for $\beta, \gamma$, and $\phi$ to minimize the influence of the prior on the posterior distribution. For the prior of $\mathrm{d} \wedge(\cdot)$, we consider the conjugate independent increments prior suggested by [26], namely $d \Lambda_{0}(t) \sim \operatorname{Gamma}\left(c \times d \Lambda_{0}^{*}(t), c\right)$, where $d \Lambda_{0}^{*}(t)$ is a prior guess for $\Lambda_{0}(t)$ and c controls the prior precision with small values of $c$ corresponding to weak prior beliefs.

\section{Selection of Biomarker Density Functions}

Since the distribution of cytokine biomarker measurements is often positively skewed and fits a lognormal distribution [27, 28], a logarithmic transformation of $g(\cdot)$ is typically used with normality assumption for $f\left(x \mid z_{i}, \phi\right)$. A QQ-plot can be used to evaluate this assumption. When the normality assumption is violated, other parametric models, such as GLMs, can be specified for the biomarkers subject to the DL and implemented easily in WinBUGS or JAGS. On the other hand, the selection of biomarker density functions can be viewed as a special case of model comparison and be investigated via Bayes factors, model diagnostics, and goodness of fit measurements [29].

\section{Simulation Studies}

We conducted extensive Monte Carlo simulations to evaluate the performance of the proposed Bayesian. We considered 18 scenarios, which included 3 distributions (normal, $t_{25}$, and gamma) for biomarker measurements, 3 percentages of measurements below the DL $(10 \%, 30 \%, 50 \%)$, and 2 coefficients $(\beta=0.8$ for true association, and $\beta=0$ for null). In addition to a biomarker, we also simulated 2 other continuous covariates according to the motivating study. We simulated both event time and censoring time with a Weibull distribution which used pre-specified baseline hazards for event and censoring and pre-specified coefficients for the biomarker and covariates. The censoring proportions of the survival time were set to $15 \%$ throughout the simulations. For each study scenario, we considered 200 subjects and 1,000 simulations. When analyzing power change for the 9 scenarios of positive association, we used $\beta=0.225$ so that the full data analysis had $80 \%$ power to reject the null hypothesis at which the biomarker values were simulated from the log-normal distribution.

In this study, we compared the following 5 methods for their performance on the estimates of regression coefficients: (1) single replacement of non-detects with one-half of the LDL threshold; (2) case-wise deletion of non-detects; (3) regular multiple imputation (MI) method; (4) extrapolation by the ROS; and (5) the proposed Bayesian approach. Single replacement with one-half $\mathrm{DL}$ or $1 / \sqrt{2}$ is the most popular method. Deletion is simply excluding (case-wise) all the 
observations with values below the threshold in the analysis. MI refers to the regular multiple imputation method assuming missing at random [30]. We modified the $\mathrm{Ml}$ procedure proposed by [31] so that the new procedure takes all aspects of uncertainty in the imputations into account [32-35]. ROS is a method which is often used in environmental science to compute summary statistics [12]. This method fits a regression line on a normal probability plot of the uncensored data, using censored values as place holders, and estimates the model parameters from the regression line, including mean and standard deviation (SD). We used the ROS-generated mean and SD to simulate values below the $\mathrm{DL}$ according to a truncated normal distribution. Cox regression was then used to estimate the hazard with the complete data set that combined the simulated values with the observed data. The simulation of the truncated normal distribution and the following Cox proportional hazards model were repeated $\mathrm{C}$ times to adjust for the uncertainty due to the simulation [36, 37]. The point estimate was the average of the $C$ imputed data sets, and the standard error (SE) was calculated using Rubin's rule [38]. In the simulation of the truncated data set, we used $C=10$.
Note that the ROS algorithm adopted here is a modified version which properly accounts for the simulation variability. The original ROS in [12] used $C=$ 1 , as the goal was to estimate summary statistics.

All computations herein were implemented using $R$ 2.15.2, and Bayesian computation was conducted using $R$ package R2jags. As both frequentist and Bayesian methods were considered in this paper, we evaluated the methods using bias, empirical SE, average SE, root mean square error (RMSE), and coverage probability of $95 \%$ confidence interval $(\mathrm{Cl})$ or Highest Posterior Density (HPD) (95\% CP), as well as power for true $\beta$ and type I error rate for null $\beta$.

\section{Simulation I}

This simulation evaluated the model performance model when the distribution of the biomarker measurements was correctly specified. Especially, we simulated the logarithmically transformed biomarker values from $\mathrm{N}(3.7,1.25)$. All the parameter estimates were computed using a multivariable Cox proportional hazards model in which no correlation was assumed for the covariates. As in Table 1, the parameter estimates

Table 1: Estimates of $\log (\mathrm{OR})$ in Simulation I

\begin{tabular}{|c|c|c|c|c|c|c|c|}
\hline \multirow[t]{2}{*}{$\mathbf{P}^{*}$} & \multirow[t]{2}{*}{ Method } & \multicolumn{5}{|c|}{$\beta_{1}=0.8$} & \multirow{2}{*}{$\begin{array}{c}\beta_{1}=0 \\
\operatorname{Err}(\%)^{\dagger \dagger}\end{array}$} \\
\hline & & Bias $^{\dagger}$ & $\mathrm{ESE}^{\ddagger}$ & $\mathrm{ASE}^{\S}$ & RMSE & $\mathrm{CP}(\%)^{* *}$ & \\
\hline \multirow[t]{6}{*}{0.1} & Full & 0.010 & 0.083 & 0.081 & 0.083 & 0.95 & 4.6 \\
\hline & Half DL & -0.037 & 0.080 & 0.078 & 0.088 & 0.92 & 4.6 \\
\hline & Deletion & 0.014 & 0.095 & 0.092 & 0.096 & 0.95 & 4.7 \\
\hline & $\mathrm{MI}$ & 0.009 & 0.106 & 0.113 & 0.107 & 0.96 & 3.8 \\
\hline & ROS & -0.031 & 0.084 & 0.087 & 0.089 & 0.93 & 4.6 \\
\hline & Bayesian & 0.011 & 0.084 & 0.082 & 0.085 & 0.94 & 4.8 \\
\hline \multirow[t]{6}{*}{0.3} & Full & 0.012 & 0.082 & 0.081 & 0.083 & 0.94 & 6.7 \\
\hline & Half DL & -0.122 & 0.091 & 0.072 & 0.152 & 0.53 & 6.0 \\
\hline & Deletion & 0.022 & 0.115 & 0.112 & 0.117 & 0.95 & 5.8 \\
\hline & $\mathrm{Ml}$ & -0.025 & 0.167 & 0.158 & 0.169 & 0.93 & 2.7 \\
\hline & ROS & -0.031 & 0.084 & 0.087 & 0.089 & 0.93 & 4.6 \\
\hline & Bayesian & 0.013 & 0.086 & 0.085 & 0.087 & 0.95 & 6.8 \\
\hline \multirow[t]{6}{*}{0.5} & Full & 0.010 & 0.082 & 0.081 & 0.083 & 0.95 & 5.3 \\
\hline & Half DL & -0.229 & 0.062 & 0.063 & 0.237 & 0.07 & 5.4 \\
\hline & Deletion & 0.025 & 0.166 & 0.161 & 0.168 & 0.96 & 5.9 \\
\hline & $\mathrm{Ml}$ & -0.141 & 0.282 & 0.250 & 0.315 & 0.85 & 3.0 \\
\hline & ROS & -0.152 & 0.078 & 0.096 & 0.171 & 0.64 & 2.3 \\
\hline & Bayesian & 0.008 & 0.097 & 0.093 & 0.098 & 0.95 & 5.9 \\
\hline
\end{tabular}

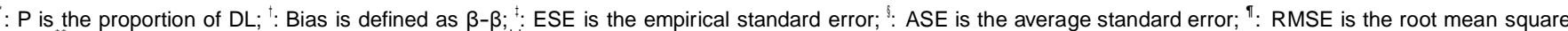
error; " : CP is the coverage probability for $95 \% \mathrm{HPD} ;{ }^{+\dagger}$ : Err is the type I error rate under null hypothesis. 


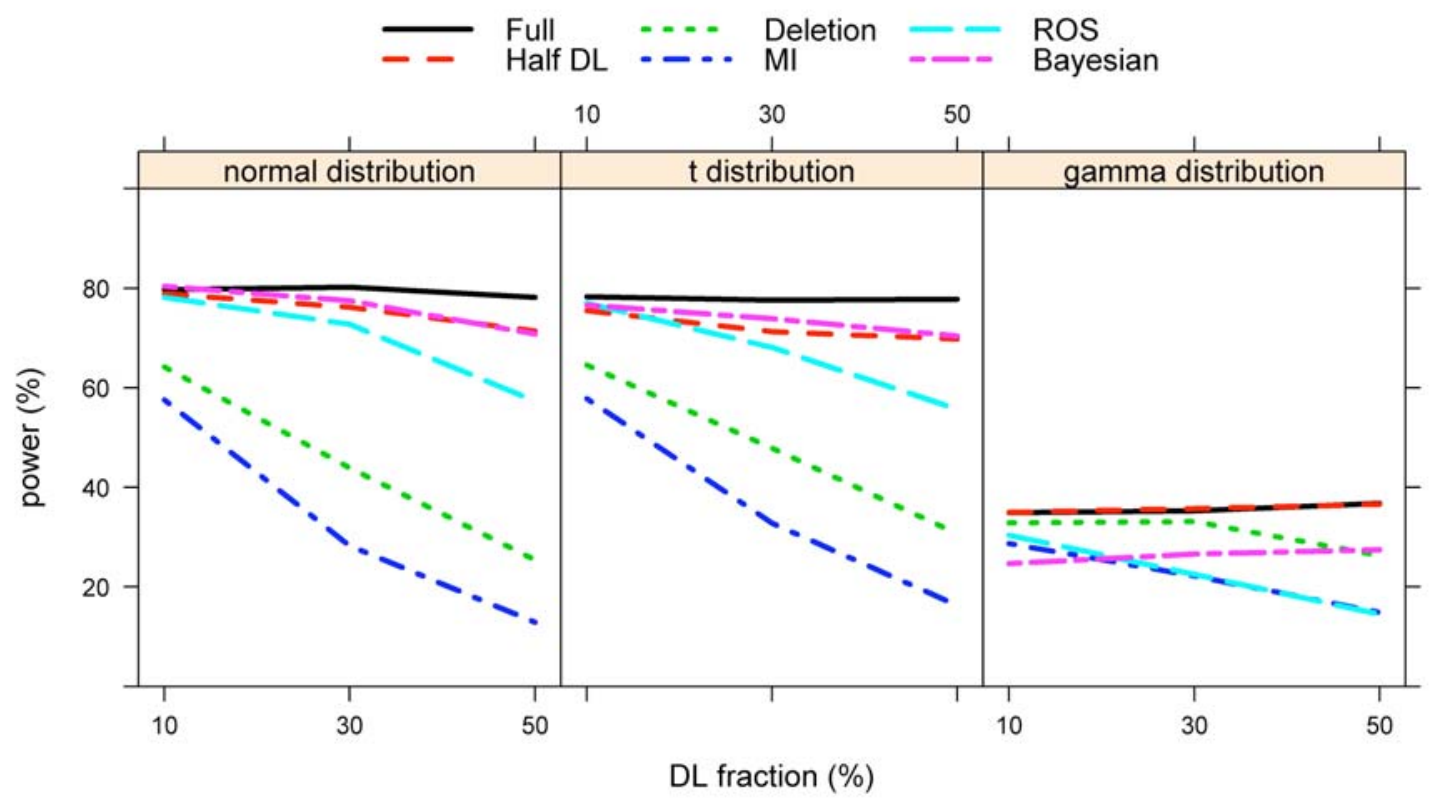

Figure 1: Power Analysis for Simulation Studies.

did not show much difference across the 5 methods at $10 \%$ LDL, but the difference became substantial at higher LDL fractions. The method of $1 / 2 \mathrm{DL}$ had the largest bias and the lowest $95 \% \mathrm{Cl}$ coverage. MI and ROS also had substantial biases, large RMSEs, and poor coverage probabilities (Table 1). Although the deletion method had negligible bias, it had a larger RMSE and poorer power compared to the Bayesian method (Table 1 and Figure 1). Overall, the Bayesian method outperformed the other methods in terms of RMSE, CP, and power.

\section{Simulation II}

We investigated the robustness of the proposed Bayesian method with respect to violation of the lognormal assumption of the biomarker measurements. We simulated the data in the same manner as in Simulation I except that the logarithmically transformed biomarker values were obtained from a heavier tailed $\mathrm{t}_{25}$ distribution with mean 3.7. The study showed similar results as Simulation I. The Bayesian method still outperformed other methods for this mild misspecification of the distribution, although its coverage probability started to fall below the nominal rate with increased DL fraction (Table 2 and Figure 1).

\section{Simulation III}

We further evaluated the proposed Bayesian method for robustness with severe misspecification of the underlying normal distribution using a highly skewed gamma distribution. We simulated the data as above, except that the logarithmically transformed biomarker was specified as a $\operatorname{Gamma}(0.5,1)$ distribution. For this serious misspecification, the proposed Bayesian method had increased bias and lower CP. As shown in Figure 2, the normality assumption is clearly violated judged from the QQ-plot against a normal distribution for logarithmic transformation of the DL biomarker variable in a randomly selected simulated data set. Interestingly, single replacement with the one-half DL method had very small bias, and the bias did not increase with higher DL fractions. This is because Gamma $(0.5,1)$ is highly positively skewed and has $10 \%, 30 \%$ and $50 \%$ threshold values of $0.008,0.074$, and 0.227 with corresponding half DL values of 0.004 , 0.037 , and 0.114 , respectively. ROS, another distribution-based method, performed the worst in this setting (Table 3 ). Although the deletion method showed merit in this scenario, its estimates had high efficiency loss. Furthermore, as shown in Figure 3 of Section 5, the deletion method did not provide valid summary statistics, such as mean or median, which is directly related to the distribution of the DL biomarker.

\section{ANALYSIS OF THE ACUTE LUNG INJURY STUDY}

The proposed method was applied to analyze data from the acute lung injury (ALI) study introduced in the Section of motivating study. In this analysis, we are interested in the association between biomarker IL8 and time to VR, controlling for age and alveolar-arterial $\mathrm{O}_{2}$ difference (A.a). We used the proposed Bayesian method to study the association between IL8 and VR 
Table 2: Estimates of $\log (\mathrm{OR})$ in Simulation II

\begin{tabular}{|c|c|c|c|c|c|c|c|}
\hline $\mathbf{P}^{*}$ & Method & \multicolumn{5}{|c|}{$\beta_{1}=0.8$} & $\begin{array}{l}\beta_{1}=0 \\
\operatorname{Err}(\%)^{\dagger}\end{array}$ \\
\hline \multirow{4}{*}{0.1} & HIf DL & -0.061 & 0.082 & 0.079 & 0.102 & 0.85 & 5.3 \\
\hline & Deletion & 0.014 & 0.098 & 0.093 & 0.099 & 0.94 & 5.6 \\
\hline & ROS & -0.007 & 0.086 & 0.083 & 0.086 & 0.95 & 5.2 \\
\hline & Bayesian & 0.004 & 0.088 & 0.083 & 0.088 & 0.94 & 5.9 \\
\hline \multirow[t]{3}{*}{0.3} & Full & 0.009 & 0.086 & 0.082 & 0.086 & 0.94 & 6.5 \\
\hline & ROS & -0.086 & 0.080 & 0.087 & 0.117 & 0.83 & 3.6 \\
\hline & Bayesian & -0.020 & 0.089 & 0.085 & 0.091 & 0.93 & 7.0 \\
\hline \multirow[t]{5}{*}{0.5} & Full & 0.010 & 0.083 & 0.082 & 0.084 & 0.95 & 5.5 \\
\hline & Half DL & -0.257 & 0.060 & 0.061 & 0.264 & 0.03 & 6.0 \\
\hline & Deletion & 0.028 & 0.154 & 0.150 & 0.156 & 0.95 & 5.7 \\
\hline & MI & -0.141 & 0.242 & 0.241 & 0.280 & 0.88 & 2.4 \\
\hline & ROS & -0.214 & 0.072 & 0.091 & 0.225 & 0.32 & 2.2 \\
\hline
\end{tabular}

Notations are same as Table 1.

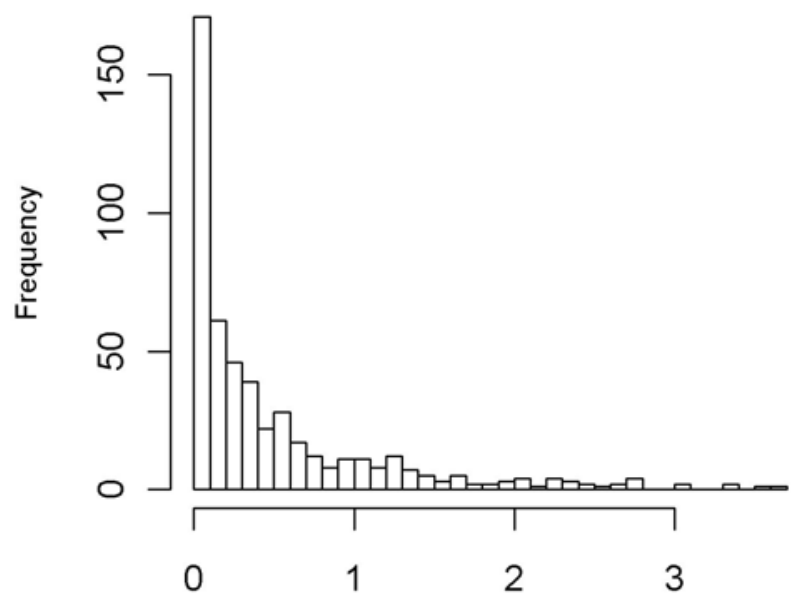

Observed biomarker value
Percent Chance of Exceedance

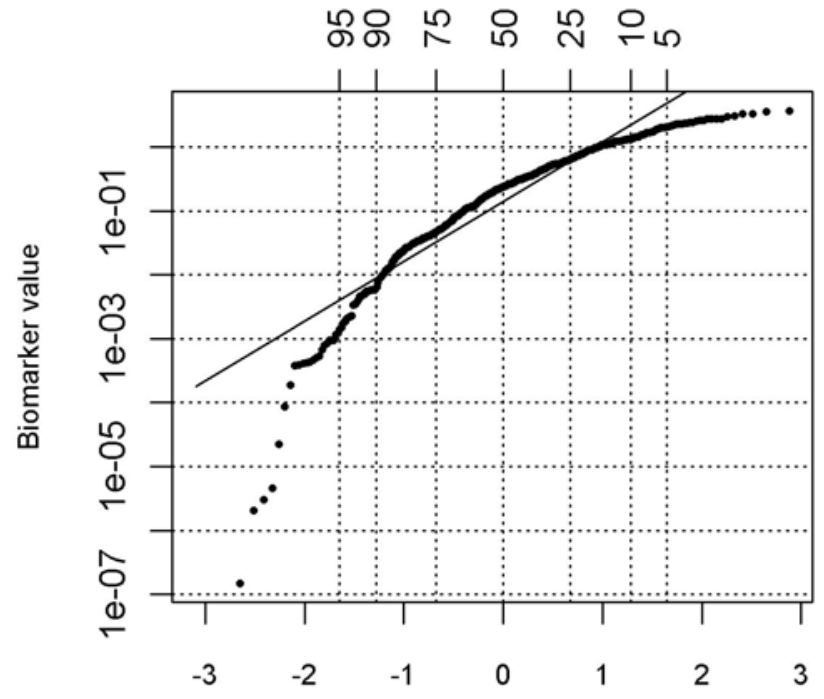

Normal Quantiles

Figure 2: Histogram Plot and QQ-plot for a Randomly Selected Dataset from Simulation III. 
Table 3: Estimates of $\log (\mathrm{OR})$ in Simulation III

\begin{tabular}{|c|c|c|c|c|c|c|c|}
\hline \multirow[t]{2}{*}{$\mathbf{P}^{*}$} & \multirow[t]{2}{*}{ Method } & \multicolumn{5}{|c|}{$\beta_{1}=0.8$} & \multirow{2}{*}{$\begin{array}{c}\boldsymbol{\beta}_{1}=0 \\
\operatorname{Err}(\%)^{\dagger}\end{array}$} \\
\hline & & Bias $^{\dagger}$ & $\mathrm{ESE}^{\ddagger}$ & ASE $^{\S}$ & RMSE $^{\prime}$ & $\mathrm{CP}(\%)^{\prime \prime}$ & \\
\hline \multirow[t]{5}{*}{0.1} & Full & 0.022 & 0.149 & 0.144 & 0.151 & 0.95 & 5.4 \\
\hline & HalfDL & 0.022 & 0.149 & 0.144 & 0.151 & 0.95 & 5.4 \\
\hline & Deletion & 0.025 & 0.155 & 0.149 & 0.157 & 0.94 & 5.6 \\
\hline & $\mathrm{Ml}$ & 0.003 & 0.153 & 0.163 & 0.153 & 0.96 & 4.6 \\
\hline & Bayesian & -0.024 & 0.147 & 0.144 & 0.149 & 0.95 & 5.6 \\
\hline \multirow[t]{4}{*}{0.3} & Full & 0.020 & 0.147 & 0.143 & 0.148 & 0.95 & 4.5 \\
\hline & Half DL & 0.023 & 0.147 & 0.143 & 0.149 & 0.95 & 4.6 \\
\hline & Deletion & 0.026 & 0.164 & 0.163 & 0.166 & 0.96 & 4.5 \\
\hline & $\mathrm{Ml}$ & -0.042 & 0.162 & 0.194 & 0.168 & 0.97 & 2.4 \\
\hline \multirow{5}{*}{0.5} & Half DL & 0.034 & 0.145 & 0.144 & 0.149 & 0.95 & 4.7 \\
\hline & Deletion & 0.034 & 0.197 & 0.188 & 0.200 & 0.95 & 5.3 \\
\hline & MI & -0.102 & 0.192 & 0.230 & 0.217 & 0.96 & 2.7 \\
\hline & ROS & -0.348 & 0.101 & 0.125 & 0.362 & 0.18 & 1.7 \\
\hline & Bayesian & -0.189 & 0.121 & 0.127 & 0.225 & 0.68 & 5.5 \\
\hline
\end{tabular}

Notations are same as Table 1.

Original data

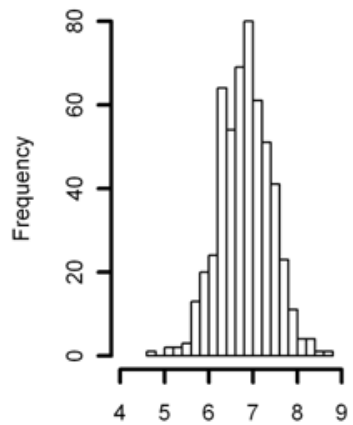

$\log (\mathrm{ICAM}-1)$

MI

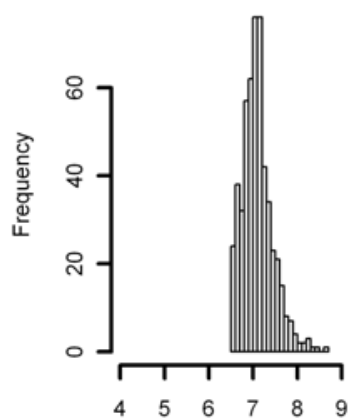

$\log (I C A M-1)$
One half DL

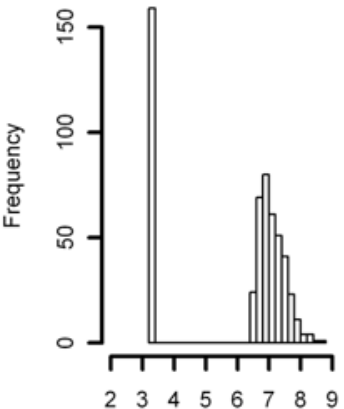

$\log (\mathrm{ICAM}-1)$

ROS

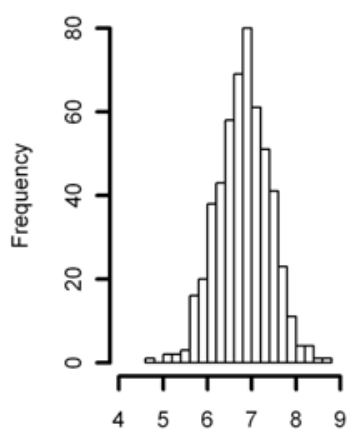

$\log (\mathrm{ICAM}-1)$
Deletion

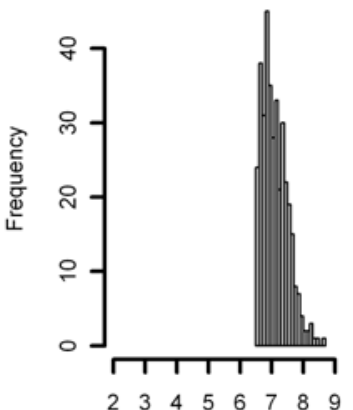

$\log (\mathrm{ICAM}-1)$

Bayesian

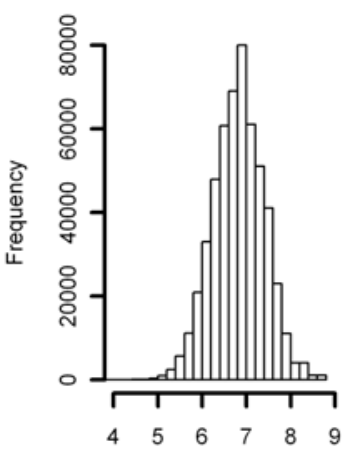

$\log (\mathrm{ICAM}-1)$

Figure 3: Histogram Plot of $\log ($ ICAM.1) in ALI Study Completed with Imputed Values. 


\section{Percent Chance of Exceedance}

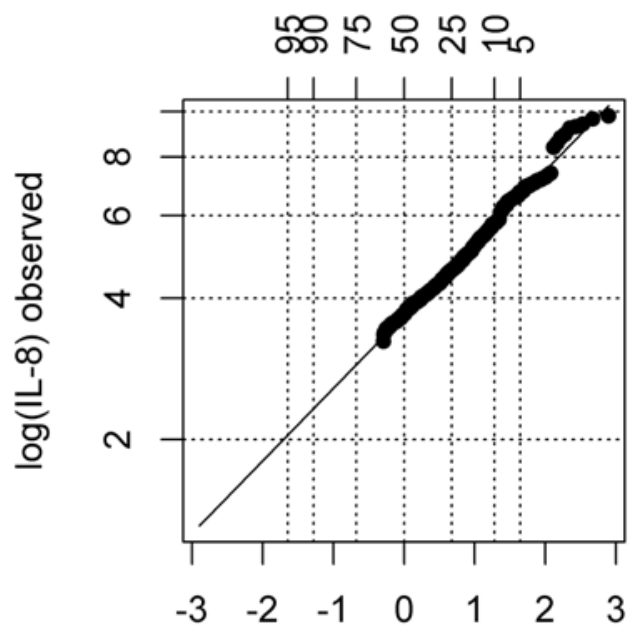

Normal Quantiles

\section{Percent Chance of Exceedance}

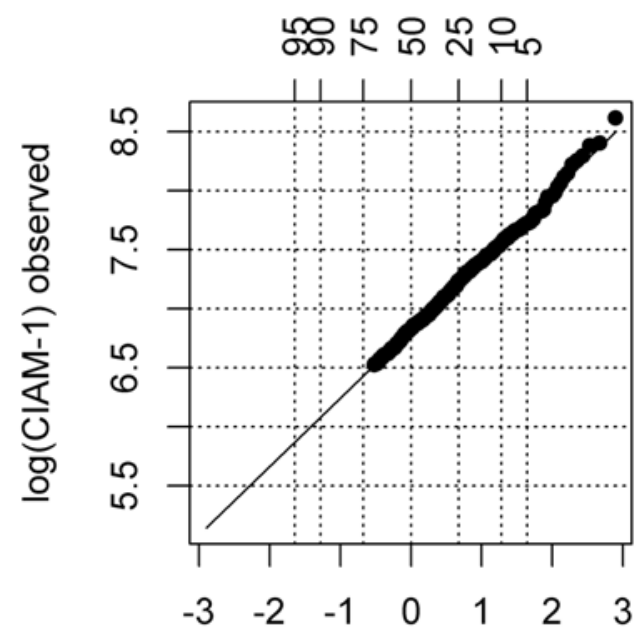

Normal Quantiles

Figure 4: QQ-plots for Logarithmic Transformation of Observed log(IL8) and $\log ($ ICAM-1) in ALI Study.

and compared the Bayesian result with the other 4 methods. A log-normal distribution seemed reasonable based on the QQ-plot of the observed data, although $38 \%$ of the IL8 data in this study are subject to the DL (Figure 4 left panel). Trace plots were used to evaluate the convergence of the parameters (not shown due to limited number of tables and figures). Based on the Cox proportional hazards model fit with the imputed data, a moderate negative association was found in the Bayesian method for IL8 with the "hazard" of removing ventilation (HR = 0.83; 95\% HPD: 0.78-0.89), suggesting the removal of ventilation was less likely for patients with higher IL8 (Single Biomarker Analysis in Table 4). The other methods yielded HR estimates ranging from 0.72 to 0.85 with larger standard error estimates compared to the Bayesian approach.
We also used the ALI study to demonstrate the methods in the scenario with more than one biomarker subject to DL. To this end, we truncated ICAM.1 to generate a dataset with $30 \%$ observations below DL. We also performed a sensitivity analysis to evaluate the impact of sequential conditioning of the two biomarkers subject to DL. In particular, we considered the Cox proportional hazards model

$\lambda[t \mid \log (I L 8), \log (I C A M .1)$, age,$A . a]=\lambda_{0}(t) \exp \left[\beta_{0}\right.$

$+\beta_{1} \log (I L 8)+\beta_{2} \log ($ ICAM.1 $)+\beta_{3}$ age $+\beta_{4}$ A.a $]$

and assume

$f(\log ($ IL8) $), \log ($ ICAM.1) age, A.a $)=f(\log ($ IL8 $)$

age, A.a, $\log ($ ICAM.1) $) f(\log ($ ICAM.1) $\mid$ age , A.a $)$,

Table 4: $\log (\mathrm{HR})$ Estimate of $\log ($ IL8) (and $\log ($ ICAM.1)) in ALI Study with Sensitivity Analysis

\begin{tabular}{|c|c|c|c|c|c|c|}
\hline \multirow[t]{3}{*}{ Method } & \multirow{2}{*}{\multicolumn{2}{|c|}{$\begin{array}{c}\text { Single Biomarker Analysis } \\
\log \left(\text { IL8) }\left(\alpha_{1}\right)\right.\end{array}$}} & \multicolumn{4}{|c|}{ Multiple Biomarkers Analysis ${ }^{\dagger}$} \\
\hline & & & \multicolumn{2}{|c|}{$\log (\operatorname{IL} 8)\left(\beta_{1}\right)$} & \multicolumn{2}{|c|}{$\log (\operatorname{ICAM} .1)\left(\beta_{2}\right)$} \\
\hline & Estimate & SE & Estimate & SE & Estimate & SE \\
\hline Half DL & -0.250 & 0.044 & -0.241 & 0.044 & -0.051 & 0.032 \\
\hline Deletion & -0.328 & 0.068 & -0.322 & 0.073 & -0.137 & 0.164 \\
\hline MI & -0.306 & 0.065 & -0.289 & 0.071 & -0.202 & 0.129 \\
\hline ROS & -0.158 & 0.033 & - & - & - & - \\
\hline Bayesian & -0.184 & 0.032 & - & - & - & - \\
\hline Bayesian1 & - & - & -0.175 & 0.035 & -0.160 & 0.100 \\
\hline Bayesian2 & - & - & -0.173 & 0.033 & -0.160 & 0.096 \\
\hline
\end{tabular}

${ }^{*}$ model is $\lambda[\mathrm{t} \mid \log (\mathrm{IL} 8)$, age, A.a $]=\lambda_{0}(\mathrm{t}) \exp \left[\alpha_{0}+\alpha_{1} \log (\mathrm{IL} 8)+\alpha_{2}\right.$ age $+\alpha_{3}$ A.a] with Bayesian method modeling $f(\log ($ IL8) $\mid$ A.a, $\log ($ ICAM.1)) with linear regression model.

tmodel is stated in (10) with Bayesian1 and Bayesian2 methods used the models stated in equations (11) and (12), respectively. 
or

$$
\begin{aligned}
& f(\log (\text { IL8) }) \log (\text { ICAM .1) age, A.a })=f(\log (\text { ICAM.1 }) \\
& \mid \text { age, A.a, } \log (\text { IL8) }) f(\log (\text { IL8) } \mid \text { age, A.a })
\end{aligned}
$$

for the joint distribution of IL8 and ICAM.1. Figure 3 shows the histograms of log(ICAM.1) that combine the observed and imputed values of each method, as well as the full original data. Only the ROS and Bayesian methods yielded distributions that are close to the original data. Recall that the ROS method did not provide valid estimates and inferences for the regression coefficient of the DL biomarker in the previous simulation studies. This observation is not surprising as the ROS method was originally proposed to estimate summary statistics, such as the mean of the biomarker, but not to estimate the regression coefficient of the DL biomarker. The log-normal assumption for ICAM.1 was evaluated by QQ-plot of the observed data (Figure 4, right panel). Table $\mathbf{4}$ shows that the Bayesian HR estimates are robust to the order of the sequential conditioning $(\mathrm{HR}=0.84$ with 95\% HPD 0.78-0.90 in Bayesian1 versus HR $=0.84$ with 95\% HPD 0.79-0.90 in Bayesian2, Multiple Biomarker Analysis in Table 4). ROS estimates were left in blank in Table 4 because the NADA package in $R$ only handles single biomarker subject to $\mathrm{DL}$.

\section{DISCUSSION}

This article proposes a general Bayesian approach for the Cox proportional hazards model with explanatory measurement variables subject to DL. We focused on the Cox proportional hazards model as it is the most widely-used model for survival analysis. The validity and application of the proposed approach do not rely on the proportional hazards assumption of the Cox model, thus, generalizing the method to other time-to-event models and incorporating a variety of techniques in Bayesian inference and diagnostics are straightforward [29]. With the counting process notation, we can extend our method to the Cox model with timedependent covariates and random effect (frailty) models for multiple event time data, among others. The JAGS code in the Appendix can be easily modified to incorporate the extension.

The proposed Bayesian method performed well when biomarker measurement distribution was correctly specified or mildly misspecified as shown in the motivating example and Simulations I and II. However, the proposed Bayesian method was not robust to severe misspecification of the underlying distribution as shown in Simulation III. Our study demonstrates the importance of an appropriate specification of DL variable distribution in improving the model performance. The QQ-plot approach or model selection criteria such as deviance information criterion can be used to guide the distribution specification in this setting. When the normality assumption is violated, other parametric models can be specified for the biomarkers subject to the DL and implemented easily in JAGS or OpenBUGS by modifying the example programs given in the Appendix. If the parametric distribution assumption is reasonable, the proposed Bayesian approach can yield valid and efficient inference with joint posterior modeling for covariates with nondetects and an outcome variable. Furthermore, in order to cope with the challenges of the common practice of the multiple biomarker approach in disease outcome prediction [19], we extended the proposed Bayesian method to the case of multiple biomarkers subject to the DL through a sequence of conditional distributions. In this situation, a sensitivity analysis needs to be considered to access the effect of the order of conditioning on the biomarkers.

\section{ACKNOWLEDGEMENTS}

The authors thank the editor and referees for helpful comments. The work received support from National Institutes of Health (R21HL097334, ULI RR024975-01, HL081332).

\section{APPENDIX}

JAGS code is provided below. Centered values are used in continuous variables.

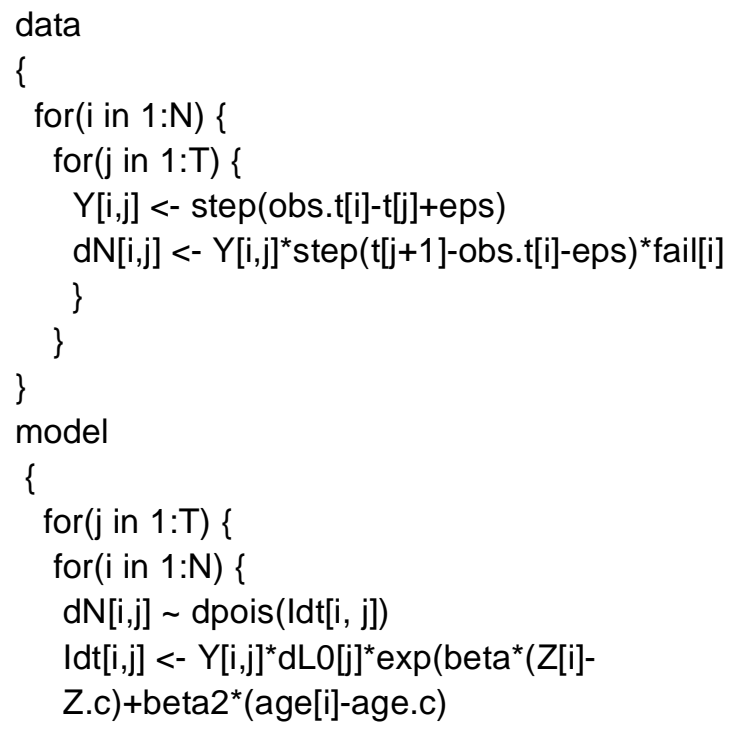




$$
\begin{aligned}
& \text { +beta3*(il8[i]-il8.c)) } \\
& \text { \} } \\
& \text { dLO[j] dgamma(mu[j],c) } \\
& \mathrm{mu}[j]<-\mathrm{dLO} \text {.star[j] }{ }^{\star} \mathrm{c} \\
& \} \\
& \text { for (i in } 1: \mathrm{N})\{ \\
& \text { cens.il8[i] dinterval(logLDL,il8[i]) } \\
& \text { il8[i] dnorm(mu.il8[i],tau) } \\
& \text { mu.il8[i] <- alpha0+alpha2* (Z[i]- } \\
& \text { Z.c)+alpha3* (age[i]-age.c) } \\
& \text { \} } \\
& c<-0.001 \\
& r<-0.1 \\
& \text { for ( } \mathrm{j} \text { in } 1: \mathrm{T}) \text { \{ } \\
& \text { dL0.star[j] }<-r^{*}(t[j+1]-t[j]) \\
& \text { \} } \\
& \}
\end{aligned}
$$

\section{REFERENCES}

[1] Baker M. In biomarkers we trust? Nature biotechnology. 2005; 23(3): 297-304 http://dx.doi.org/10.1038/nbt0305-297

[2] Ray P, et al. Statistical evaluation of a biomarker. Anesthesiology 2010; 112(4): 1023-40. http://dx.doi.org/10.1097/ALN.0b013e3181d47604

[3] Morikawa $\mathrm{T}$, et al. Association of CTNNB1 ( $\beta$-catenin) alterations, body mass index, and physical activity with survival in patients with colorectal cancer. JAMA: the journal of the American Medical Association 2011; 305(16): 1685-94. http://dx.doi.org/10.1001/jama.2011.513

[4] García-Bilbao A, et al. Identification of a biomarker panel for colorectal cancer diagnosis. BMC Cancer 2012; 12(1): 43.

[5] Braunwald E. Biomarkers in heart failure. New Engl J Med 2008; 358(20): 2148-59.

http://dx.doi.org/10.1056/NEJMra0800239

[6] Patel DD. Prognostic significance of immunohistochemically localized biomarkers in stage II and stage III breast cancer: a multivariate analysis. Ann Surg Oncol 2000; 7(4): 305-11. http://dx.doi.org/10.1007/s10434-000-0305-5

[7] McShane LM, et al. Reporting recommendations for tumor marker prognostic studies (REMARK). J Natl Cancer Instit 2005; 97(16): 1180-84. http://dx.doi.org/10.1093/jnci/dji237

[8] Hall P, Going J. Predicting the future: a critical appraisal of cancer prognosis studies. Histopathology 1999 35(6): 48994.

http://dx.doi.org/10.1046/j.1365-2559.1999.00862.x

[9] Cochran WG. Errors of measurement in statistics. Technometrics 1968; 10(4): 637-66.

http://dx.doi.org/10.2307/1267450

[10] Carroll RJ, et al. Measurement error in nonlinear models: a modern perspective. 2010: CRC press.

[11] Schisterman EF, et al. The limitations due to exposure detection limits for regression models. Am J Epidemiol 2006; 163(4): 374-83. http://dx.doi.org/10.1093/aje/kwj039

[12] Helsel DR. Nondetects and data analysis. Statistics for censored environmental data. 2005: Wiley-Interscience.

[13] Cole SR, et al. Estimating the odds ratio when exposure has a limit of detection. Int J Epidemiol 2009; 38(6): 1674-80. http://dx.doi.org/10.1093/ije/dyp269 subject to a detection limit. Epidemiology (Cambridge, Mass.) 2010; 21(Suppl 4): S17. http://dx.doi.org/10.1097/EDE.0b013e3181ce97d8

Henschel V, et al. A semiparametric Bayesian proportional hazards model for interval censored data with frailty effects. BMC Med Res Methodol 2009; 9(1): 9.

Wang HJ, Feng X. Multiple Imputation for M-Regression With Censored Covariates. J Am Statist Assoc 2012; 107(497): 194-204.

http://dx.doi.org/10.1080/01621459.2011.643198

$\mathrm{Wu} \mathrm{H}$, et al. A Bayesian approach for generalized linear models with explanatory biomarker measurement variables subject to detection limit: an application to acute lung injury. $J$ Appl Statist 2012; 39(8): 1733-47. http://dx.doi.org/10.1080/02664763.2012.681362

Dagne GA, Huang Y. Bayesian semiparametric mixture Tobit models with left censoring, skewness, and covariate measurement errors. Statist Med 2013.

Fellahi J-L, et al. Simultaneous Measurement of Cardiac Troponin I, B-type Natriuretic Peptide, and C-reactive Protein for the Prediction of Long-term Cardiac Outcome after Cardiac Surgery. Anesthesiology 2009; 111(2): 250-57. http://dx.doi.org/10.1097/ALN.0b013e3181a1f720

] Ware LB, et al. Prognostic and pathogenetic value of combining clinical and biochemical indices in patients with acute lung injury. CHEST J 2010; 137(2): 288-96. http://dx.doi.org/10.1378/chest.09-1484

] Cox DR. Regression models and life-tables. Journal of the Royal Statistical Society. Series B (Methodological) 1972; 187-220.

Ibrahim JG, Lipsitz SR, Chen MH. Missing covariates in generalized linear models when the missing data mechanism is non-ignorable. Journal of the Royal Statistical Society: Series B (Statistical Methodology) 1999; 61(1): 173-90. http://dx.doi.org/10.1111/1467-9868.00170

Chen Q, Ibrahim JG. Semiparametric models for missing covariate and response data in regression models. Biometrics 2006; 62(1): 177-84.

http://dx.doi.org/10.1111/j.1541-0420.2005.00438.x

Clayton DG. A Monte Carlo method for Bayesian inference in frailty models. Biometrics 1991; 467-485.

\section{http://dx.doi.org/10.2307/2532139}

Andersen PK, Gill RD. Cox's regression model for counting processes: a large sample study. Ann Statist 1982; 11001120.

http://dx.doi.org/10.1214/aos/1176345976

6] Kalbfleisch JD. Non-parametric Bayesian analysis of survival time data. Journal of the Royal Statistical Society. Series B (Methodological) 1978; 214-221.

7] Koch AL. The logarithm in biology 1. Mechanisms generating the log-normal distribution exactly. J Theoret Biol 1966; 12(2): 276-90.

http://dx.doi.org/10.1016/0022-5193(66)90119-6

8] Limpert E, Stahel WA, Abbt M. Log-normal distributions across the sciences: keys and clues. BioScience 2001; 51(5): 341-52.

http://dx.doi.org/10.1641/00063568(2001)051\%5B0341:LNDATS\%5D2.0.CO;2

[29] Ibrahim JG, Chen MH, Sinha D. Bayesian survival analysis. 2005: Wiley Online Library.

[30] Rubin DB. Inference and missing data. Biometrika 1976; 63(3): 581-92. http://dx.doi.org/10.1093/biomet/63.3.581

[31] Lubin $\mathrm{JH}$, et al. Epidemiologic evaluation of measurement data in the presence of detection limits. Environ Health Perspect 2004; 112(17): 1691. 
[32] De Groot J, et al. Multiple imputation to correct for partial verification bias revisited. Statist Med 2008; 27(28): 5880-89. http://dx.doi.org/10.1002/sim.3410

[33] Harrell FE. Regression modeling strategies: with applications to linear models, logistic regression, and survival analysis. 2001: Springer.

[34] Little R, Hyonggin A. Robust likelihood-based analysis of multivariate data with missing values 2003 .

[35] Siddique J, Belin TR. Multiple imputation using an iterative hot-deck with distance-based donor selection. Statist Med 2008; 27(1): 83-102. http://dx.doi.org/10.1002/sim.3001
[36] Hopke PK, Liu C, Rubin DB. Multiple Imputation for Multivariate Data with Missing and Below-Threshold Measurements: Time-Series Concentrations of Pollutants in the Arctic. Biometrics 2001; 57(1): 22-33. http://dx.doi.org/10.1111/j.0006-341X.2001.00022.x

[37] Uh H-W, et al. Evaluation of regression methods when immunological measurements are constrained by detection limits. BMC Immunol 2008; 9(1): 59. http://dx.doi.org/10.1186/1471-2172-9-59

[38] Rubin DB. Multiple imputation for nonresponse in surveys. 2009; Vol. 307. Wiley. com.

(C) 2014 Chen et al.; Licensee Lifescience Global.

This is an open access article licensed under the terms of the Creative Commons Attribution Non-Commercial License (http://creativecommons.org/licenses/by-nc/3.0/) which permits unrestricted, non-commercial use, distribution and reproduction in any medium, provided the work is properly cited. 